\title{
Efficient Integration of Sampling-Based Spatial Conditional Failure Joint Probability Densities
}

\author{
Michael P. Enright \\ Southwest Research Institute, San Antonio, TX, 78238, USA \\ Harry R. Millwater ${ }^{\dagger}$, and Jonathan P. Moody ${ }^{\ddagger}$ \\ University of Texas at San Antonio, San Antonio, TX, 78249, USA
}

Joint probability density functions (JPDFs) can be used to describe the likelihood of spatial position or events that are dependent on spatial position. Event-based JPDFs are often based on computational outcomes at specific locations, where the number of potential locations may be unlimited. The efficiency and accuracy associated with the estimation of the conditional failure JPDF is dependent on the number of spatial locations and the number of limit state evaluations at each spatial location. An approach is presented for the estimation of a fracture mechanics-based conditional failure JPDF within a finite domain. An approximate JPDF is constructed using probability of fracture values associated with anomalies placed at discrete points along the perimeter of the component and at selected locations within the interior of the component. The discrete points are connected to form a mesh of elements, and anomaly occurrence rates are assigned to elements based on their relative volumes. The final JPDF is obtained from an adaptive refinement of the element mesh where elements are subdivided based on contribution to component risk. The results can be applied to the risk assessment of components that are susceptible to fracture failure.

\section{Nomenclature}

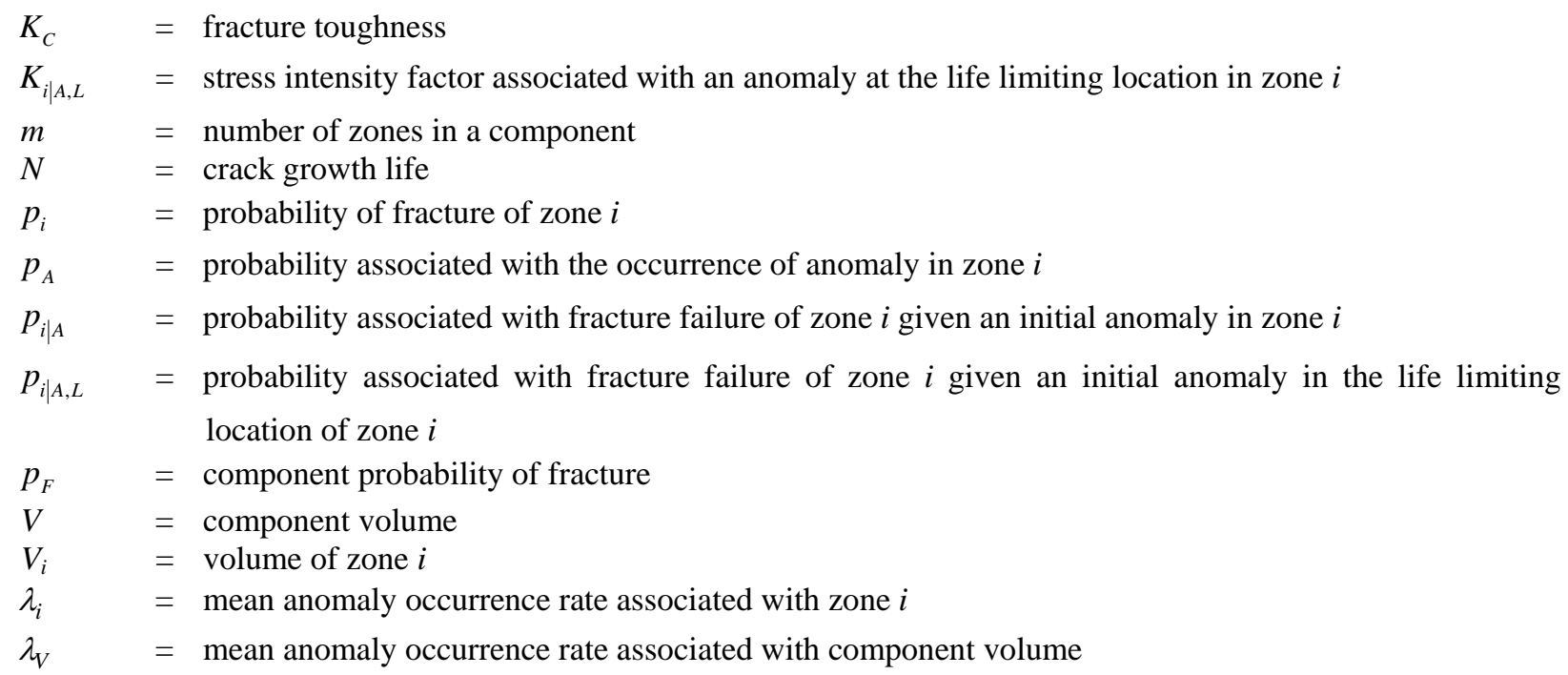

\section{Introduction}

Toint probability density functions are commonly used to describe the occurrence frequency associated with multiple random variables. If sufficient data are available, they can be used to describe the likelihood of spatial

\footnotetext{
* Principal Engineer, Reliability and Materials Integrity, 6220 Culebra Road, Member AIAA.

${ }^{\dagger}$ Associate Professor, Mechanical Engineering and Biomechanics, 6900 N. Loop 1604 West, Member AIAA.

‡ Graduate Student, Mechanical Engineering and Biomechanics, 6900 N. Loop 1604 West.
} 
position $^{1-3}$ (random variable basis), or events that are dependent on spatial position ${ }^{4,5}$ (event basis). Randomvariable-based joint probability density functions (JPDFs) are typically constructed using parametric statistical models to fit the available (but often limited) data. On the other hand, event-based JPDFs are often based on computational outcomes at specific locations, where the number of potential locations is potentially unlimited ${ }^{2}$. For example, an event-based JPDF can be used to describe the conditional probability of failure as a function of position (Fig. 1). The efficiency and accuracy of the conditional failure JPDF is dependent on the number of limit state evaluations at each spatial location and on the number of spatial locations used to describe it. Component failure probability $\left(p_{F}\right)$ is obtained by integrating the spatial conditional failure JPDF over the spatial domain, where computational efficiency and accuracy is dependent on the underlying conditional failure JPDF at the selected integration points.

Previous studies ${ }^{6-8}$ have focused on modeling the spatial conditional failure JPDF as a multivariate histogram associated with regions within the domain that are referred to as "zones". The uniform probability density for each zone is set to the maximum possible value within the associated region to ensure a conservative value of $p_{F}$. The advantage to this approach is that a relatively coarse mesh can be used to estimate conditional failure JPDF, and the mesh can be refined as necessary to satisfy computational accuracy requirements. The primary disadvantage to this approach is that it requires definition of the maximum conditional failure probability within each zone. In addition, the mesh refinement process may require computation of conditional failures at more locations than would be needed to accurately describe the spatial conditional failure JPDF.

In this paper, an approach is presented for the estimation of a fracture mechanics-based conditional failure JPDF within a finite domain. An approximate JPDF is constructed using probability of fracture values associated with anomalies placed at discrete points along the perimeter of the component and at selected locations within the interior of the component. The discrete points are connected to form a mesh of elements using a Delaunay triangulation algorithm $^{9,10}$. Anomaly occurrence rates are assigned to elements based on the relative values of the element volumes. The final JPDF is obtained from an adaptive refinement of the element mesh where elements are subdivided based on contribution to component risk.

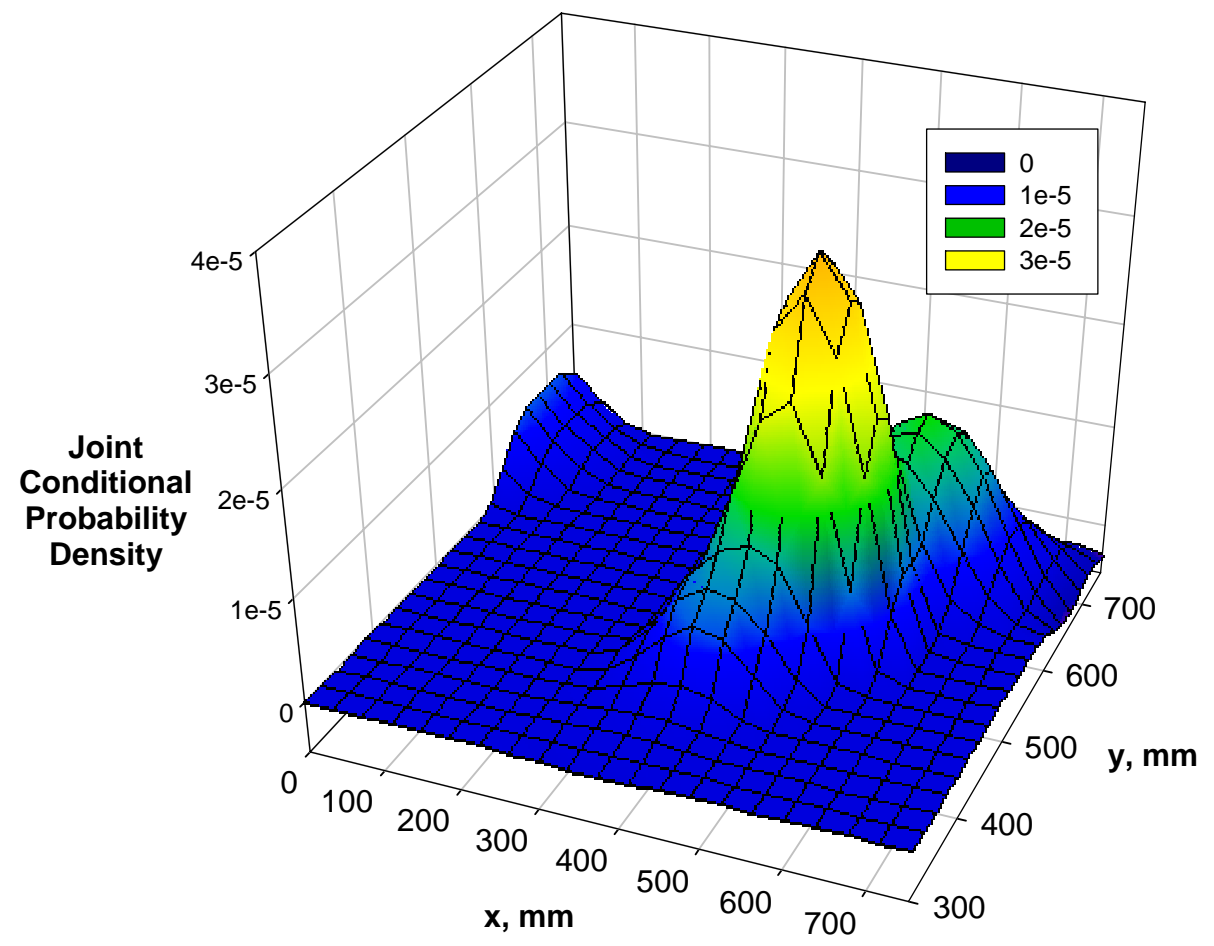

Figure 1. A joint probability density function (JPDF) can be used to describe the conditional probability of failure as a function of position. 


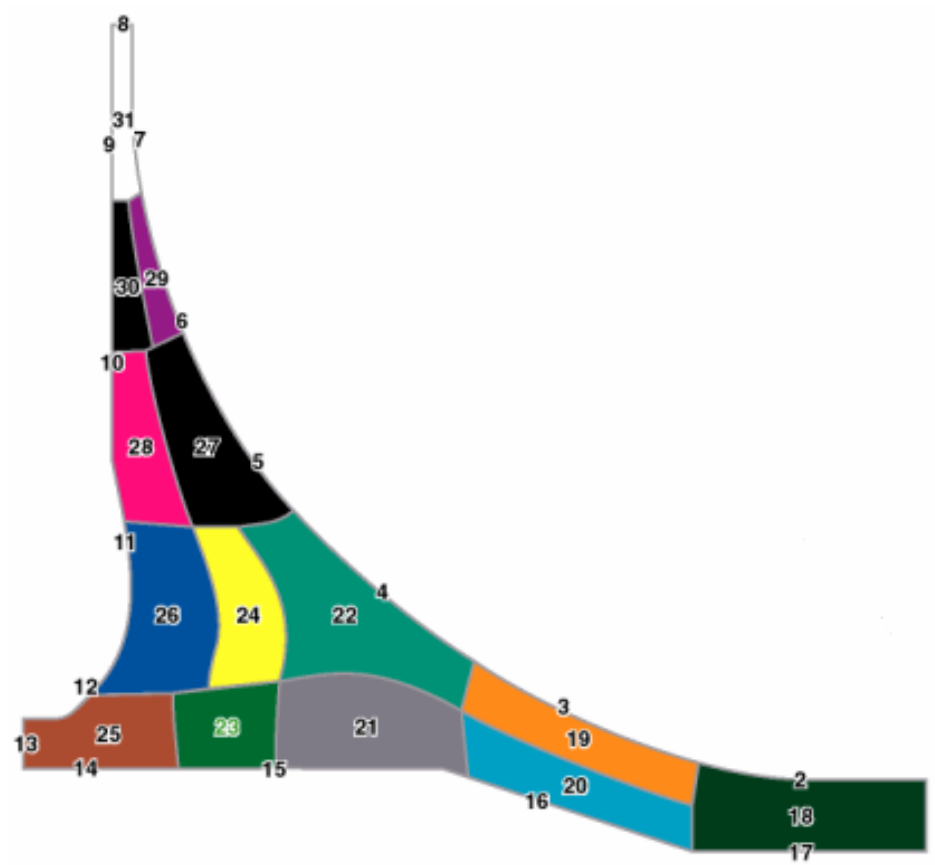

Figure 2. The conditional failure JPDF can be approximated as a histogram using a zone-based approach.

\section{Zone-Based Probabilistic Fracture Mechanics}

The computation time associated with a probabilistic fracture mechanics-based risk assessment may be nontrivial. To improve the efficiency of risk computations, an approximate solution can be used to address the uncertainty associated with the location of the anomaly. As shown in Fig. 2, the disk is subdivided into regions of approximately equal risk, called zones. It is assumed that all of the material in a zone has similar stresses, material properties, inspection schedules and POD, and the crack growth life associated with an initial anomaly is similar anywhere within a zone. For rare anomaly materials it is assumed that a zone has no more than one significant anomaly. To ensure a conservative risk estimate, the anomaly is placed in a location within the zone that minimizes the crack growth life values.

For rare anomaly materials, the probability of fracture $p_{i}$ within a zone is given by ${ }^{6}$ :

$$
p_{i}=p_{i \mid A} \cdot p_{A}
$$

$p_{A}$ can be modeled as a Poisson process ${ }^{2}$ with a mean anomaly occurrence rate $\lambda_{i}$ that is proportional to the volume of material in the zone:

$$
\lambda_{i}=\frac{V_{i}}{V} \lambda_{V}
$$

If the mean anomaly occurrence rate is relatively small, then the component probability of fracture can be approximated $\mathrm{as}^{11}$ :

$$
p_{F} \simeq \sum_{i=1}^{m} p_{i}
$$

Some materials may exhibit large anomaly occurrence rates, with multiple anomalies within a zone. If there are no physical interactions among anomalies in a zone, then the zone risk can be estimated by assuming that all anomalies are at the life limiting location of the zone. This provides a conservative (upper bound) risk estimate that converges to the true solution as the number of zones becomes large ${ }^{12}$. For materials with large numbers of anomalies, the component probability of fracture becomes ${ }^{13}$ :

$$
p_{F}=1-\prod_{i=1}^{m} \exp \left[-\lambda_{i} \cdot p_{i \mid A, L}\right]
$$


where zone failure within a occurs when the stress intensity factor at the life limiting location $K_{i \mid A, L}$ exceeds the fracture toughness $K_{C}$ :

$$
p_{i \mid A, L}=P\left(K_{i \mid A, L}>K_{C}\right)
$$

\section{Efficient Risk Prediction}

Sampling-based probabilistic analysis methods can be used to predict the probability of fracture of components subjected to periodic inspection. Monte Carlo simulation provides accurate results, where accuracy is dependent on the relative values of failure probability, confidence interval, and number of random samples. However, when Monte Carlo simulation is used, a time consuming crack growth assessment must performed for each Monte Carlo sample. A large number of samples may be required to satisfy accuracy requirements, so the total CPU time required to complete the computations may be significant.

To reduce computation time, a response surface can be developed that relates the fracture mechanics-based input random variables such as initial crack size and crack growth life scatter to the response variables such as final crack size and cycles to failure. During Monte Carlo sampling, estimates for the response variables are obtained from the response surface which can significantly improve computational efficiency.

Importance sampling (IS) can also be applied to the problem by focusing the analysis on the initial conditions that would result in lives shorter than the specified design life. This approach reduces the size of the analysis region and may be significantly more efficient than Monte Carlo simulation. An overview of the IS methodology for predicting fatigue life of components subjected to periodic inspections is provided in references 6 and 14. A summary of some recently developed techniques for efficient risk assessment of fracture critical components subjected to random inspection is provided in Table 1.

\section{Estimation of Conditional Failure Joint Probability Density}

As an alternative to the zone-based approach, a conditional failure JPDF can be used to represent the probability of failure given spatial position within a domain. Conditional failure probabilities are typically estimated at discrete locations to describe a joint probability mass function (JPMF) over the domain. Since the JPDF is a continuous function, some form of response surface must be developed to transform the discrete JPMF into a continuous function. If a coarse mesh is used to estimate the JPMF, it may introduce considerable error into the JPDF. On the other hand, if a fine mesh is used, the computation time required to develop the JPMF may be prohibitive, particularly when a numerical sampling-based approach is used to estimate failure probabilities. JPDF estimation is therefore focused on the creation of the mesh of discrete points that are used to describe the JPMF.

As indicated in Eqn. (1), the probability of fracture given an initial anomaly is a conditional failure probability that is dependent on the size and location of the anomaly within a component. The probability of fracture is dependent on the applied stress values and on the geometry of the component (among other factors), both of which are dependent on the location of the anomaly. Probability of fracture values are greatest in regions of relatively high stress and low constraint, which frequently occurs on or near the component surface. Mesh points are placed along the surface of the component to model the potentially large conditional failure values in this region. These points can be joined together, and provide a description of the component perimeter or exterior boundary. Additional mesh points can be added to the interior to address the changes in probability of fracture values associated with these

Table 1. Efficient computational techniques for risk prediction of fracture critical components subjected to random inspection

\begin{tabular}{llll} 
Technique & Classification & Description & Reference \\
\hline $\begin{array}{l}\text { Life Approximation } \\
\text { Function }\end{array}$ & $\begin{array}{l}\text { Tailored response } \\
\text { surface }\end{array}$ & $\begin{array}{l}\text { Create tables of crack area and cycles to failure for } \\
\text { Importance Sampling }\end{array}$ & 6,15 \\
& $\begin{array}{l}\text { Zone-based variance } \\
\text { reduction } \\
\text { Znone Refinement }\end{array}$ & $\begin{array}{l}\text { Combined technique of numerical integration and } \\
\text { failure region-based Monte Carlo simulation }\end{array}$ & 6,14 \\
Uncertainty & $\begin{array}{l}\text { Focused discretization of zones based on relative } \\
\text { contribution to component risk }\end{array}$ & 12 \\
Optimal Sampling & $\begin{array}{l}\text { Component-based } \\
\text { variance reduction }\end{array}$ & $\begin{array}{l}\text { Allocate Monte Carlo samples to each zone based on } \\
\text { its relative contribution to component risk } \\
\text { Distributed }\end{array}$ & Parallel processing \\
Processing & $\begin{array}{l}\text { Provide computational framework for distributing } \\
\text { computational tasks to multiple computers }\end{array}$ & 18 \\
\hline
\end{tabular}


locations. A Delaunay triangulation algorithm is used to combine the perimeter and interior points into a triangular mesh of elements. Once the initial mesh has been formed, it can be refined using an adaptive algorithm ${ }^{18}$.

\section{Application to Gas Turbine Engine Components}

The approach for estimation of the conditional failure JPDF is illustrated for the aircraft gas turbine engine compressor disk shown in Fig. 2a. Internal stresses and temperatures are based on finite element analysis results associated with four critical load steps in the flight cycle. The disk has a design life of 20,000 flight cycles. Deterministic crack growth life was computed using stress intensity factor solutions for cracks in rectangular plates $^{19}$. Probability of fracture values were computed using importance sampling ${ }^{6,14}$.

Normalized stress values associated with the primary cycle and conditional risk values associated with all cycles are shown in Fig. 4a for each of the discrete locations along the perimeter of the component (Fig. 3b). Perimeter nodes were placed at locations of significant change in stress. Conditional risk values were computed at these locations, also shown in Fig. 4a, where it can be observed that the conditional risk contour is remarkably similar to the conditional risk contour associated with the discrete locations (Fig. 3b). The number and location of the perimeter nodes were adjusted using an adaptive refinement algorithm ${ }^{18}$. The conditional risk results associated with the refined mesh are shown in Fig. 4b.

The number and location of the perimeter nodes can also be adjusted so that the perimeter mesh geometry is consistent with the finite element geometry, as shown in Fig. 5. The perimeter mesh points were connected with interior mesh points using Delaunay triangulation to form the complete mesh shown in Fig. 6. The mesh was adaptively refined by subdividing the elements that have a significant contribution to risk, shown in Fig. 7. The resulting mesh (Fig 7d) includes both coarse and fine regions, reflecting relatively small and large changes in conditional failure associated with change in position, respectively. A comparison of JPDFs based on a highly discretized mesh and the adaptively refined mesh is shown in Fig 8, where similar results were obtained for both meshes.

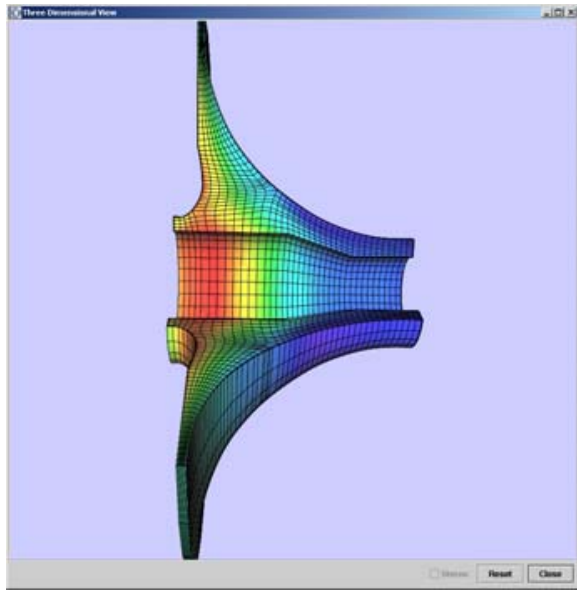

(a)

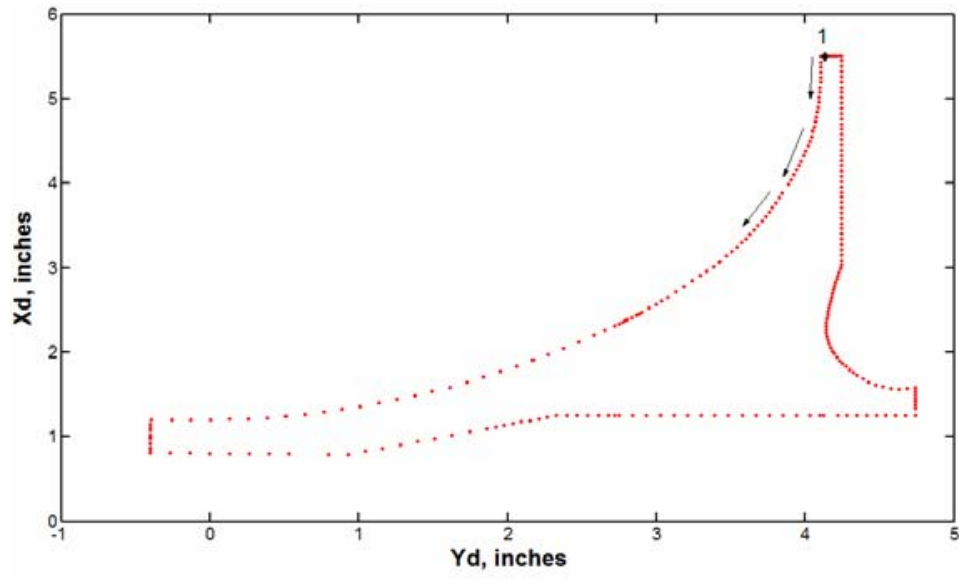

(b)

Figure 3. Conditional failure JPDF estimation of a gas turbine engine disk: (a) finite element geometry, and (b) finite element nodes associated with the surface of the axisymmetric cross section of the disk. 


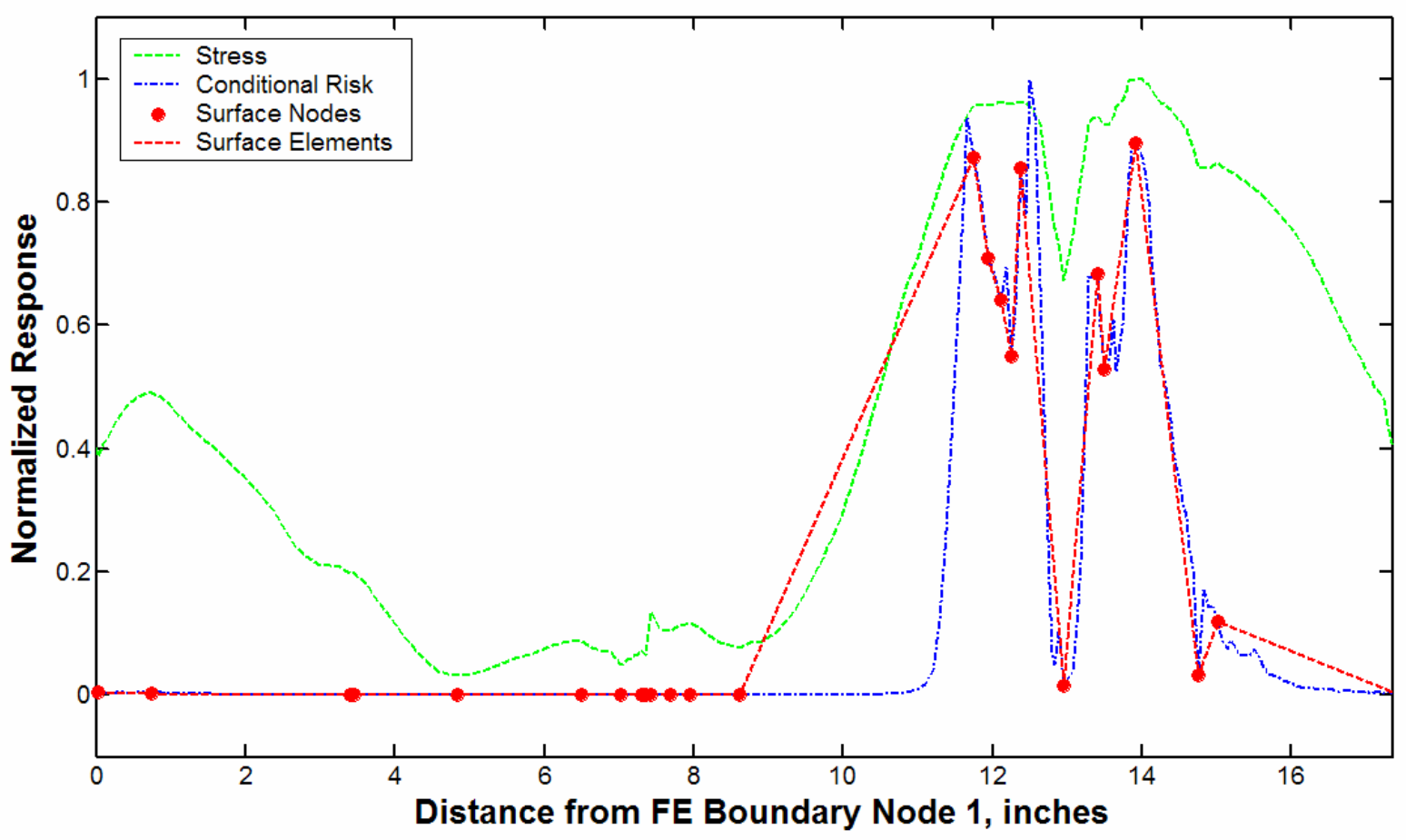

(a)

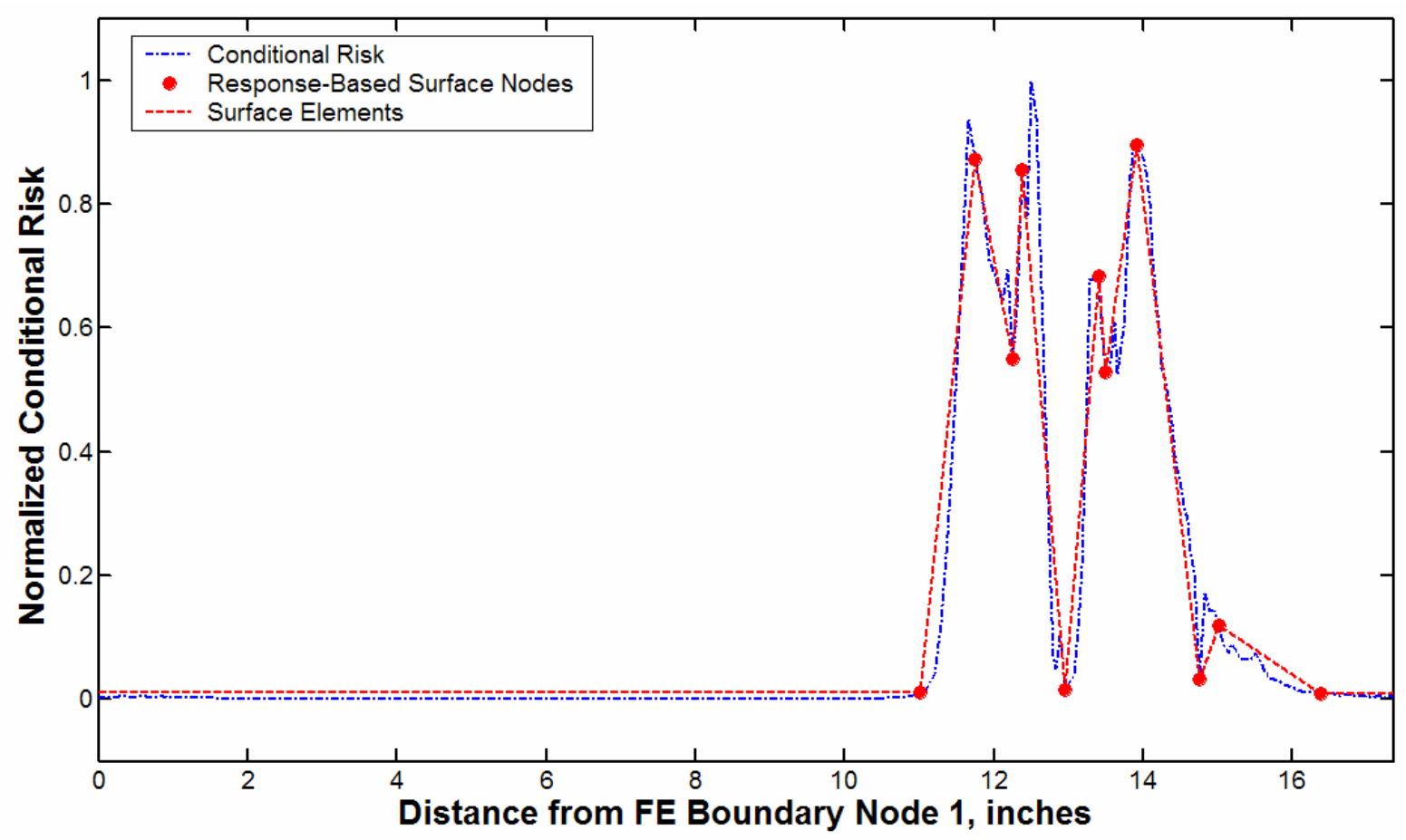

(b)

Figure 4. Normalized response values along the perimeter of the component: (a) initial perimeter mesh, (b) adaptively refined perimeter mesh. 


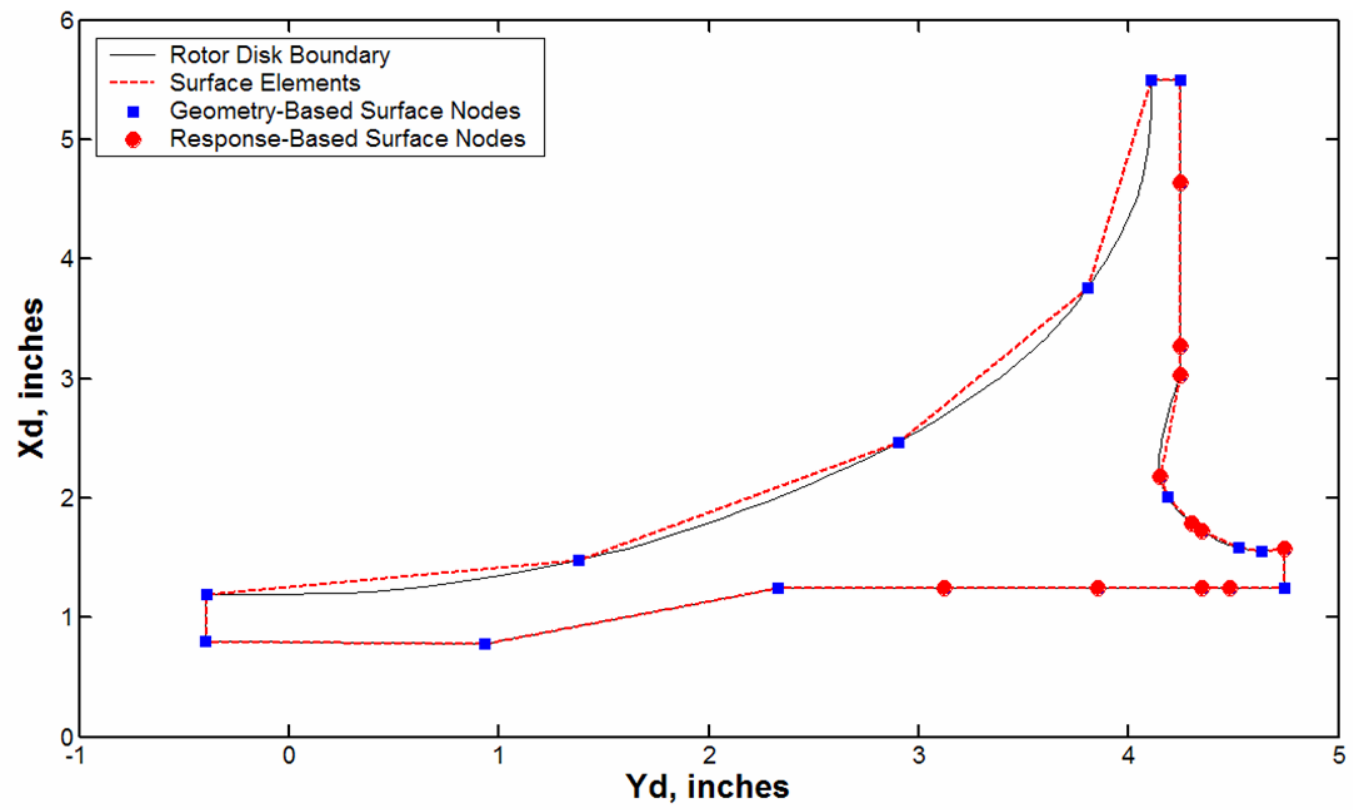

Figure 5. The number and spacing of mesh points is adjusted so that the perimeter mesh geometry is consistent with the finite element geometry.

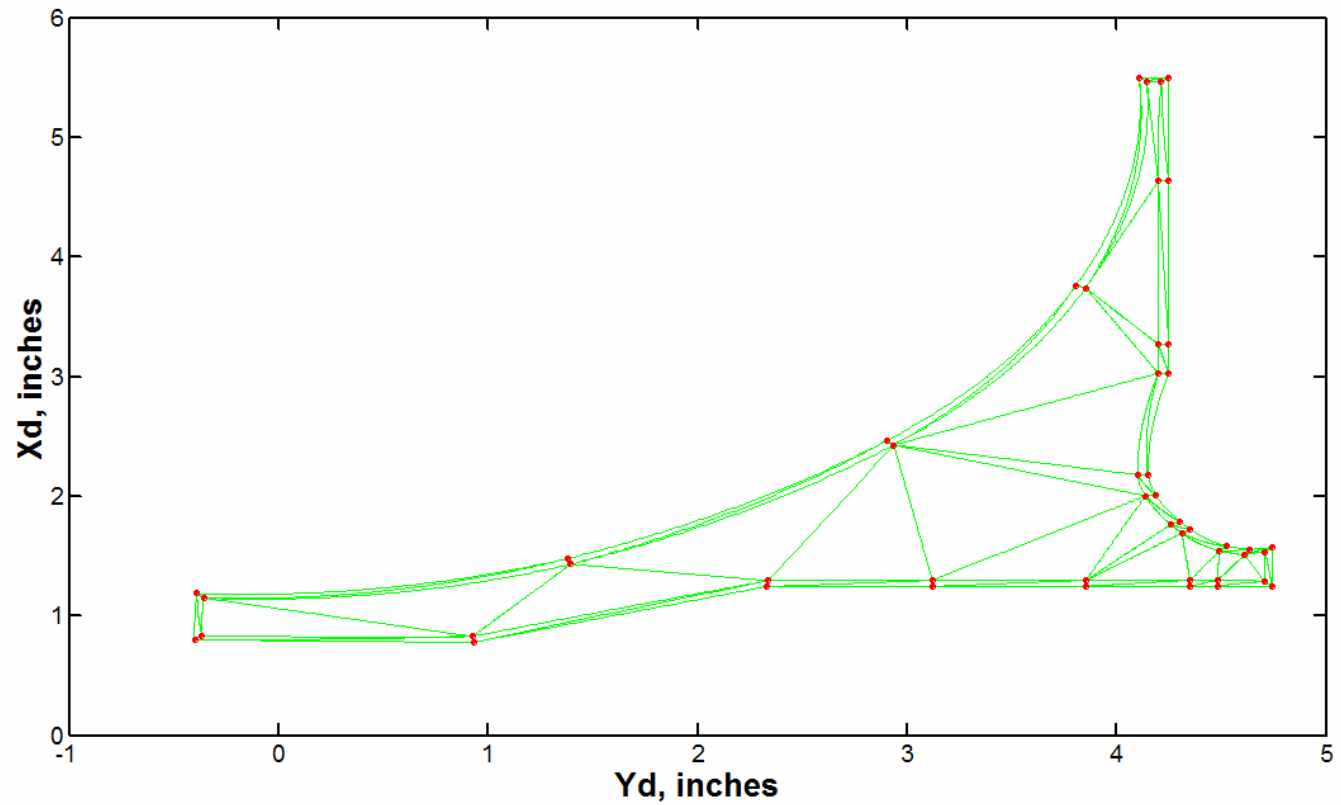

Figure 6. The perimeter mesh points are connected to interior points using a Delaunay triangulation algorithm. 


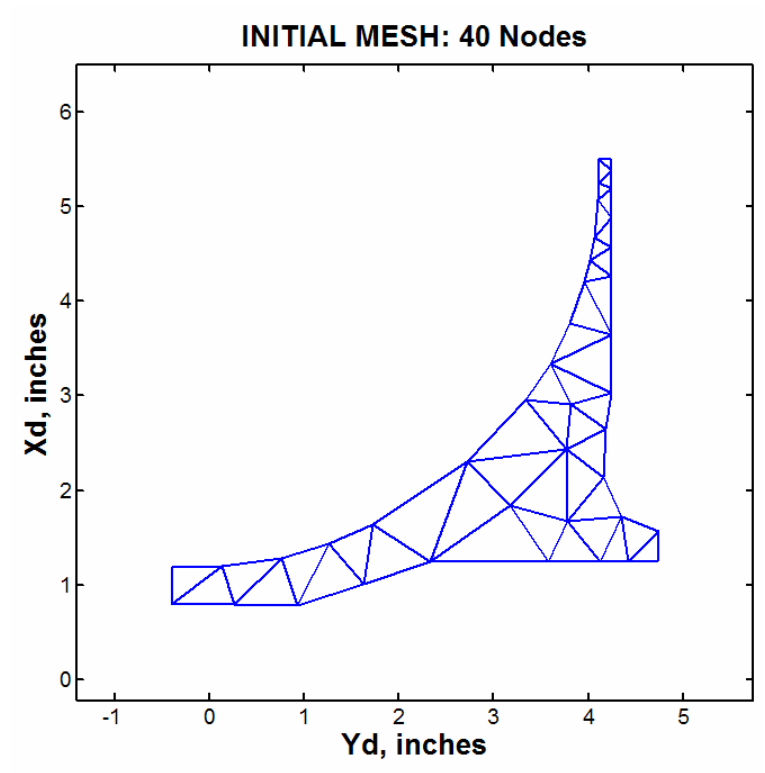

(a)

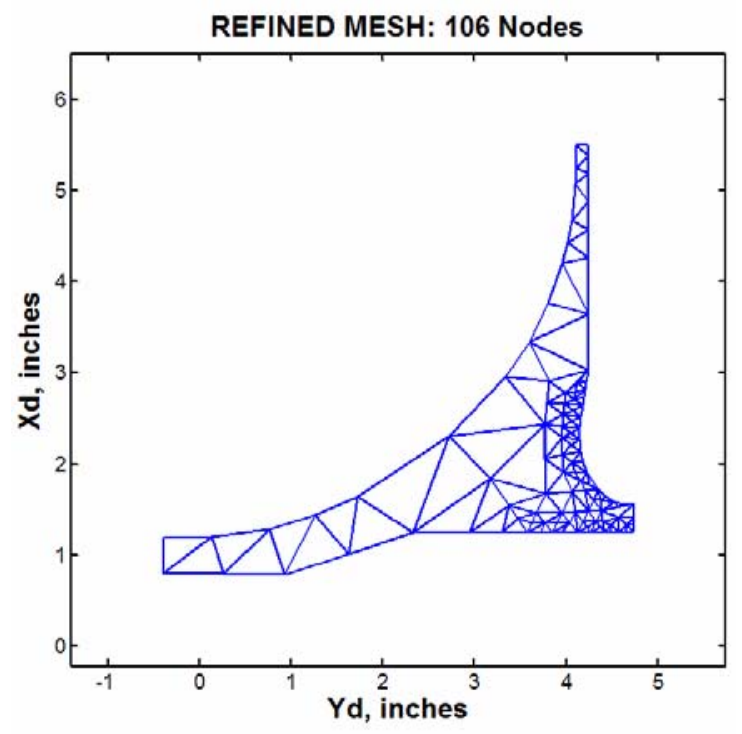

(c)

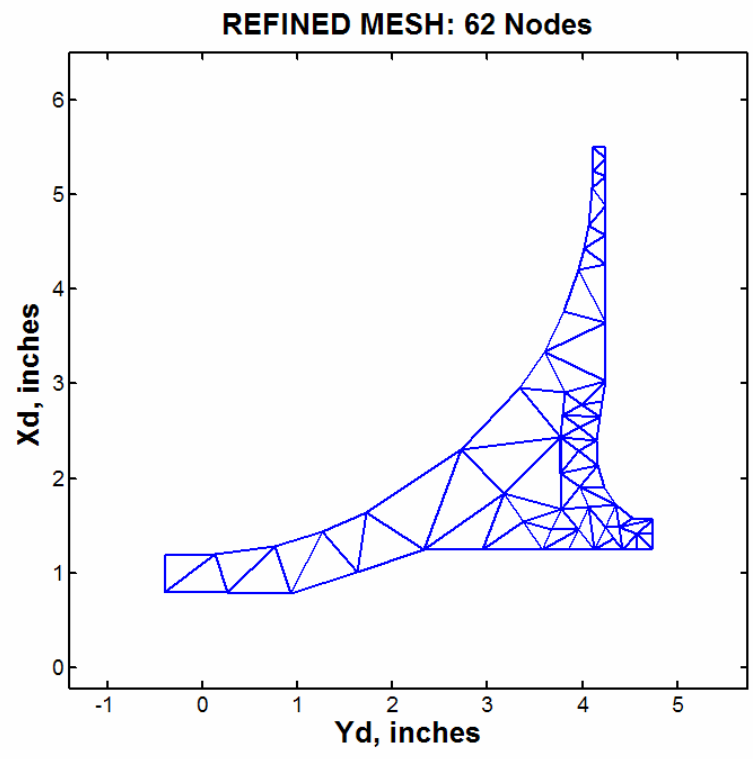

(b)

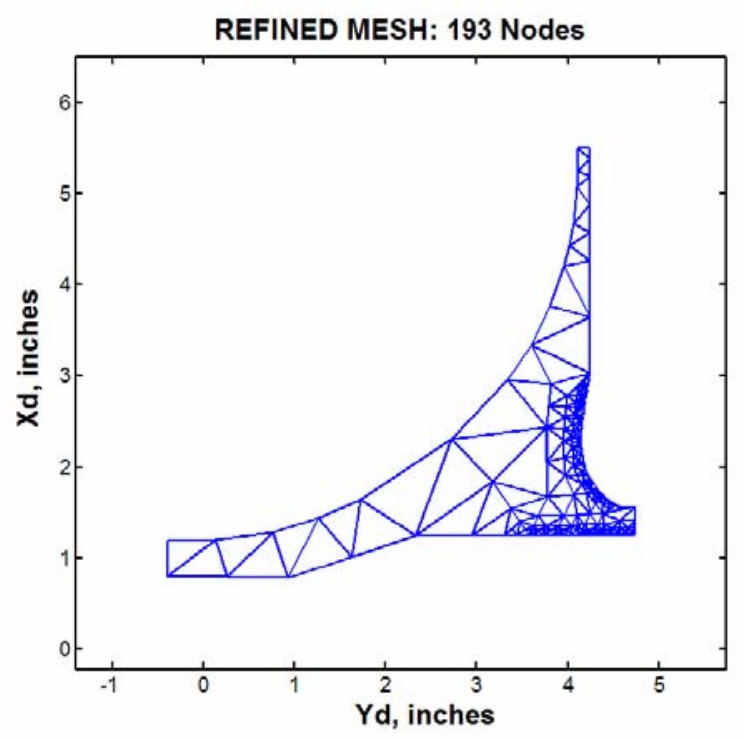

(d)

Figure 7. The mesh is adaptively refined until component risk convergence is achieved: (a) initial mesh, (b) 62 nodes, (c) 106 nodes, and (d) 193 nodes. 


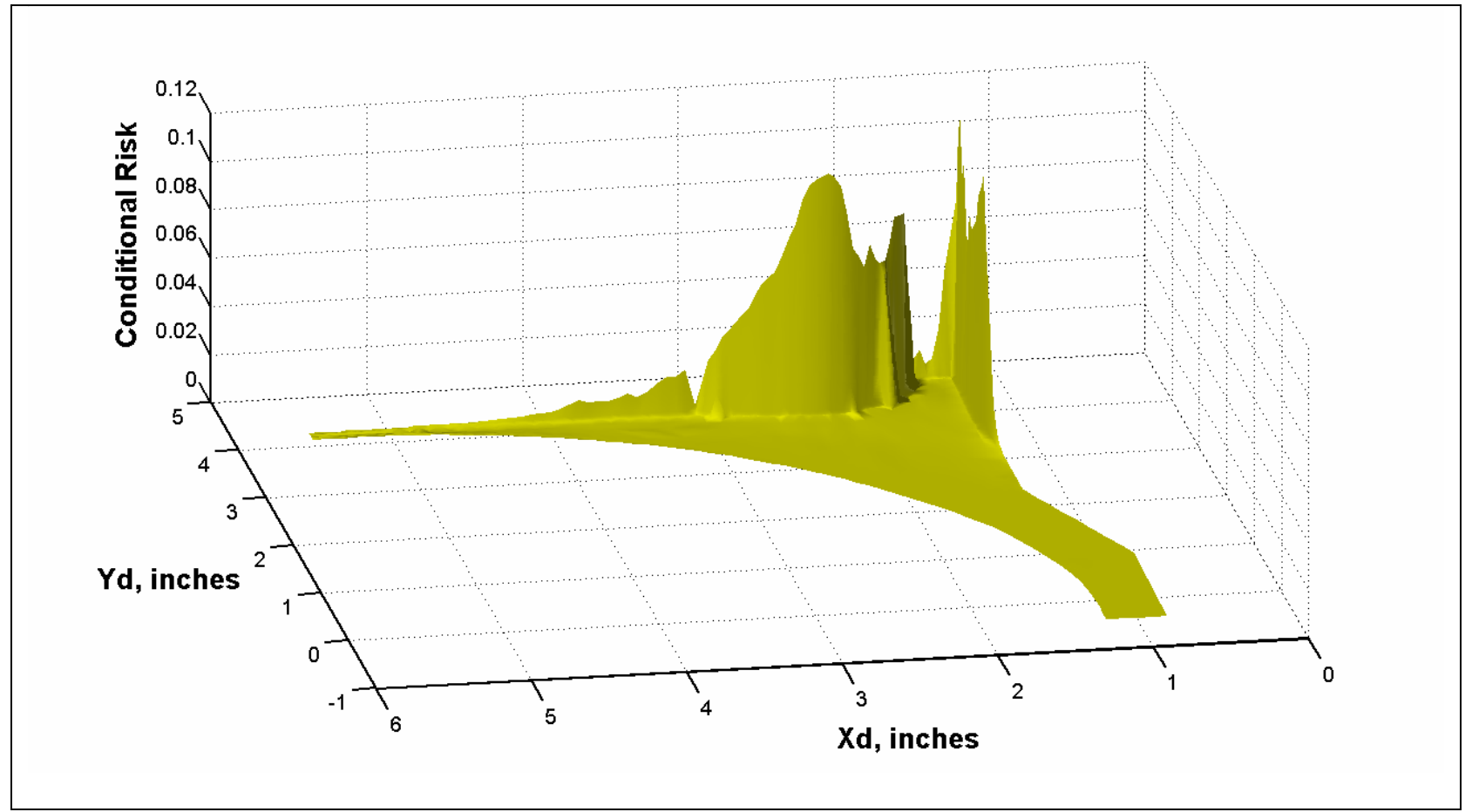

(a)

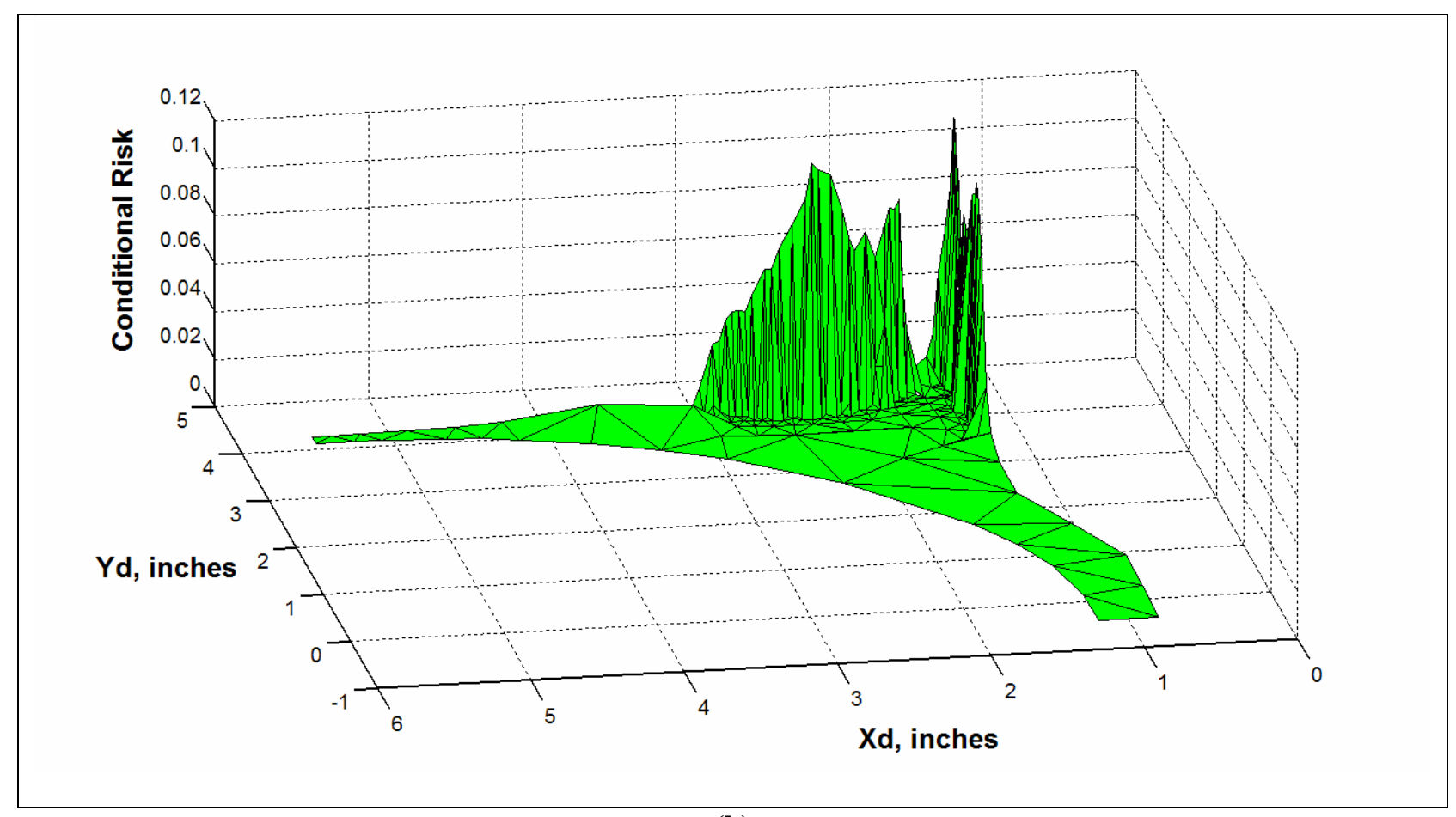

(b)

Figure 8. Comparison of conditional failure JPDFs associated with fracture of a gas turbine engine disk: (a) highly discretized parametric mesh (reference solution), and (b) adaptive refinement using 193 nodes. 


\section{Summary}

An approach was presented for the estimation of a fracture mechanics-based conditional failure JPDF within a finite domain. The approach consists of the development of an initial mesh based on discrete points along the perimeter of the component and at selected locations within the interior of the component. The final JPDF is obtained from an adaptive refinement of the element mesh where elements are subdivided based on contribution to component risk. The approach was illustrated for fracture assessment of a gas turbine engine disk. The results can be applied to the risk assessment of components that are susceptible to fracture failure.

\section{Acknowledgments}

This work was supported by the Federal Aviation Administration under Grant 2005-G-005 (Probabilistic Design for Rotor Integrity). The authors wish to thank Joe Wilson (FAA William J. Hughes Technical Center project manager) and Tim Mouzakis (FAA Engine and Propeller Directorate, New England Regional Office) for their continued support. The ongoing contributions of the Industry Steering Committee (Darryl Lehmann, Pratt \& Whitney; Bob Maffeo, General Electric; Ahsan Jameel, Honeywell; Jon Dubke, Rolls-Royce) are also gratefully acknowledged.

\section{References}

${ }^{1}$ Ang, A. H-S., and Tang, W.H., Probabilistic Concepts in Engineering Planning and Design. John Wiley, New York, 1975.

${ }^{2}$ Haldar, A. and Mahadevan, S., Probability, Reliability, and Statistical Methods in Engineering Design, John Wiley, New York, 2000.

${ }^{3}$ Ayyub, B.M., and McCuen, R.H., Probability, Statistics, and Reliability for Engineers and Scientists, Chapman \& Hall, New York, 2003.

${ }^{4}$ Scott, D.W., Multivariate Density Estimation, John Wiley, New York, 1992.

${ }^{5}$ Enright, M.P., McClung, R.C., Hudak, S.J., and Millwater, H.R., “Application of Nonparametric Methods to Rainflow Stress Density Estimation of Gas Turbine Engine Usage,” Paper GT2006-90780, Proceedings of the 51st ASME International Gas Turbine \& Aeroengine Technical Congress, ASME, Barcelona, Spain, May 8-11, 2006.

${ }^{6}$ Wu, Y.T., Enright, M.P., and Millwater, H.R., "Probabilistic Methods for Design Assessment of Reliability with Inspection,” AIAA Journal , Vol. 40, No. 5, 2002, pp. 937-946.

${ }^{7}$ Leverant, G.R., "Turbine Rotor Material Design - Final Report," DOT/ FAA/ AR-00/64, Federal Aviation Administration, Washington, DC, 2000.

${ }^{8}$ McClung, R.C., Enright, M.P., Lee, Y-D., Huyse, L., and Fitch, S.H.K, “Efficient Fracture Design for Complex Turbine Engine Components,” Journal of Engineering for Gas Turbines and Power, ASME, (accepted).

${ }^{9}$ Fernandez, F.A., Y.C. Yong and R.D. Ettinger, “A Simple Adaptive Mesh Generator for 2-D Finite Element Calculations,” IEEE Transactions on Magnetics, Vol. 29, No. 2, 1993, pp. 1882-1885.

${ }^{10}$ Shewchuk, J.R., "Delaunay Refinement Algorithms for Triangular Mesh Generation,” Computational Geometry: Theory and Applications, Vol. 22, No. 1-3, 2002, pp. 21-74.

${ }^{11}$ Freudenthal, A.M., Garrelts, J.M, and Shinozuka, M., "The Analysis of Structural Safety," Journal of the Structural Division, ASCE, Vol. 92, No. ST1, 1966, pp. 267-325.

${ }^{12}$ Millwater, H.R., Enright, M.P., and Fitch, S.H.K., “A Convergent Zone-Refinement Method for Risk Assessment of Gas Turbine Disks Subject to Low-Frequency Metallurgical Defects,” Journal of Engineering for Gas Turbines and Power, ASME, (accepted).

${ }^{13}$ Enright, M.P., and Huyse, L., "Methodology for Probabilistic Life Prediction of Multiple Anomaly Materials," AIAA Journal, AIAA, Vol. 44, No. 4, 2006, pp. 787-793.

${ }^{14}$ Huyse, L., and Enright, M.P., "Efficient Statistical Analysis of Failure Risk in Engine Rotor Disks using Importance Sampling Techniques,” Proceedings 44th Structures, Structural Dynamics, and Materials Conference, Norfolk, VA, April 7-10, 2003, AIAA Paper 2003-1838.

${ }^{15}$ Southwest Research Institute, “DARWIN ${ }^{\circledR}$ User’s Guide”, Southwest Research Institute, San Antonio, TX, 2007.

${ }^{16}$ Enright, M.P. and Millwater, H.R., "Optimal Sampling Techniques for Zone-Based Probabilistic Fatigue Life Prediction,” Proceedings of the 43rd AIAA/ASME/ASCE/AHS/ASC Structures, Structural Dynamics, and Materials Conference, NonDeterministic Approaches Forum, Denver, CO, April 22-25, 2002, AIAA Paper 2002-1383.

${ }^{17}$ Enright, M.P., Millwater, H.R., and Huyse, L., “Adaptive Optimal Sampling Methodology for Reliability Prediction of Series Systems,” AIAA Journal, AIAA, Vol. 44, No. 3, 2006, pp. 523-528.

${ }^{18}$ Moody, J.P., Millwater, H.R., and Enright, M.P., “Automatic Risk Assessment Methodology for Gas Turbine Engines Employing Adaptive Mesh Refinement Techniques,” Paper GT2007-27576, Proceedings of the 52nd ASME International Gas Turbine \& Aeroengine Technical Congress, ASME, Montreal, Canada, May 14-17, 2007.

${ }^{19}$ McClung, R.C., Enright, M.P., Millwater, H.R., Leverant, G.R., and Hudak, S.J., “A Software Framework for Probabilistic Fatigue Life Assessment,” Journal of ASTM International, Vol. 1, No. 8, 2004, Paper ID JAI11563. Also published in Probabilistic Aspects of Life Prediction, ASTM STP 1450, 2004, pp. 199-215. 